\title{
The Effects of Fibromyalgia Syndrome on Physical Function and Psychological Status of Pregnant Females
}

\author{
Hakan GENÇ, ${ }^{1}$ Melahat ATASEVER, ${ }^{2}$ Burcu DUYUR ÇAKIT, ${ }^{1}$ Murat SEVAL, ${ }^{3}$ Acar KOÇ \\ ${ }^{1}$ Department of Physical Medicine and Rehabilitation, University of Health Sciences, Ankara Training and Research Hospital, Ankara, Turkey \\ ${ }^{2}$ Department of Gynecology and Obstetric, Medical Faculty of Giresun University, Giresun, Turkey \\ ${ }^{3}$ Department of Gynecology and Obstetric, Medical Faculty of Ankara University, Ankara, Turkey
}

\begin{abstract}
Objectives: This study aims to investigate the frequency and most common symptoms of fibromyalgia syndrome (FS) among pregnant females and determine the impacts of FS on physical functioning and psychological status.

Patients and methods: A total of 360 pregnant females (mean age 26.5 years, range 19 to 42 years) were included. The subjects were divided into two groups in terms of having (FS group; $n=136$; mean age 27 years; range 19 to 41 years) or not having FS (control group; $n=224$; mean age 26.5 years; range 20 to 42 years). The impact of FS on physical functions was evaluated using Fibromyalgia Impact Questionnaire. Psychological statuses of the subjects were evaluated using State-Trait Anxiety Inventory, Wijma Delivery Expectancy/Experience Questionnaire, and Beck Depression Inventory. Results: Low back pain was the most common complaint while fatigue was the most common symptom in FS group. FS group had higher levels of pain and physical disability $(p<0.001)$ and also higher values of anxiety, fear of childbirth, and depression $(p<0.001$, for all values) compared to control group. Symptom severity and physical function scores were significantly correlated with increased levels of pain, depression, anxiety, and fear of childbirth ( $<<0.001$, for all values).

Conclusion: Fibromyalgia syndrome is common among pregnant females. The existence of FS in pregnancy is a severe factor contributing to maternal stress, anxiety, and depression. Therapeutic measures for fibromyalgia syndrome should be well-established to support healthy pregnancy and good child health outcome.

Keywords: Fibromyalgia syndrome; physical function; pregnancy; psychological status.
\end{abstract}

During pregnancy, more problems may arise particularly in the third trimester, one of them being chronic pain. Most females experience some kind of pain during pregnancy, either as a result of pre-existing conditions such as low back pain (LBP), headache or rheumatic diseases, or as a direct consequence of pregnancy such as weight gain, postural changes, pelvic floor dysfunction, or hormonal factors. ${ }^{1}$ Pregnancy-related LBP is reported as a common complaint among pregnant females with an incidence of 25 to $90 \% .^{2}$

Fibromyalgia syndrome (FS) is a common disease characterized by widespread musculoskeletal pain in certain anatomic locations. ${ }^{3} \mathrm{FS}$ is much more common in females than in males with a proportion of 9:1. Compared to males, females experience significantly more fatigue, morning fatigue, pain all over body, and irritable bowel syndrome with higher number of symptoms. Females have significantly more tender points. ${ }^{4}$ FS is seen mainly in females of reproductive age. However, the relationship between FS and pregnancy has not been wellestablished. One recent study has described a high prevalence of symptoms characteristic of the FS among healthy pregnant females. ${ }^{5}$ In another study based on retrospective interviews, most FS patients described a worsening of FS symptoms during pregnancy. ${ }^{6}$ 
There are many unexplored issues related to the presence of FS in pregnancy such as prevalence of FS, most common symptoms, the impact of FS on physical functioning, psychological status, and treatment of a fibromyalgic pregnant. So, in this study, we aimed to investigate the frequency and most common symptoms of FS among pregnant females and determine the impacts of FS on physical functioning and psychological status.

\section{PATIENTS AND METHODS}

A total of 438 pregnant females (mean age 27 years, range 19 to 43 years) in the third trimester of their pregnancy were screened for this study between March 2015 and September 2015 at Ankara University Hospital. Individuals with active inflammatory/infectious disease (e.g., rheumatoid arthritis, psoriatic arthritis, ankylosing spondylitis, acute bacterial, fungal or viral infections), active psychiatric disease, and history of previous traumatic injury within 10 days, fracture and/or dislocation, as well as high-risk pregnancies (potential complications that could affect the mother and/or the baby including advanced maternal age, using illegal drugs, underlying chronic conditions such as diabetes, high blood pressure and epilepsy, and pregnancy complications such as problems with the uterus, cervix or placenta) were excluded. Thirty-eight patients refused to participate in the study while 40 met the exclusion criteria and were excluded. Finally, a total of 360 pregnant females (mean age 26.5 years, range 19 to 42 years) were included. This study was approved by the Ankara University, Faculty of Medicine Ethics Committee and all the participants provided written informed consents prior to the study. The study was conducted in accordance with the principles of the Declaration of Helsinki.

The subjects were divided into two groups in terms of having (FS group; $n=136$; mean age 27 years; range 19 to 41 years) or not having FS (control group; $\mathrm{n}=224$; mean age 26.5 years; range 20 to 42 years). The presence of fibromyalgia was investigated via 2010 American College of Rheumatology classification criteria. ${ }^{12}$ Demographic characteristics of all subjects including age, weight, height, smoking habits, and number of pregnancy were noted. Widespread
Pain Index (WPI) and symptom severity (SS) scale scores were recorded. ${ }^{7}$ Most common pain locations and most common symptoms of FS were also noted.

Pain severity was assessed using visual analog scale (VAS). For this assessment, subjects were informed about the meaning of the numbers placed over a $10 \mathrm{~cm}$ long line. "No pain" was defined as "0" and "most severe pain" was defined as "10". Satisfaction with marriage was also evaluated using a 0-10 VAS. "No satisfaction" was defined as "0" while "being most satisfied" was defined as "10".

The impact of FS on physical functions was evaluated using the Turkish version of Fibromyalgia Impact Questionnaire (FIQ). ${ }^{8}$ The FIQ is an assessment instrument developed to measure the status, progress, and outcomes of FS patients. ${ }^{9}$ The FIQ is composed of 10 items. The first item contains 11 questions related to physical functioning; each question is rated on a 4-point Likert-type scale. The 11 questions are scored and summed to yield one physical impairment score (PIS). An average raw score between 0 and 3 is obtained. ${ }^{10}$ Items 2 and 3 ask the patients to mark the number of days they felt well and the number of days they were unable to work (including housework) because of fibromyalgia symptoms. Items 4 through 10 are horizontal linear scales marked in increments of 10 on which the patients rate work difficulty, pain, fatigue, morning tiredness, stiffness, anxiety, and depression. The FIQ is scored in such a way that a higher score indicates greater impact of the syndrome on the person. Each of the 10 items has a maximum possible score of 10 . Thus the maximum possible score is 100 . An average FS patient scores about 50, while severely afflicted patients usually score 70 or higher.

Anxiety of subjects was evaluated using the Turkish edition of State-Trait Anxiety Inventory (STAI). ${ }^{11}$ The STAI is a psychological inventory based on a 4-point Likert-type scale, consisting of 40 questions answered on a self-report basis. ${ }^{12}$ The STAI measures two types of anxiety. The first 20 questions comprise the state anxiety inventory, which includes 10 items that describe negative mood and 10 items that describe positive mood. The remaining 20 questions constitute the trait anxiety inventory, which is used to evaluate 
the frequent emotional experience of individuals. The positive mood items are reversely scored, and the total scores of state and trait anxiety inventories are calculated separately. The highest and lowest scores that can be achieved for this scale are 80 and 20, respectively. Higher scores are positively correlated with higher levels of anxiety.

Fear of childbirth both during pregnancy and after delivery was measured by the Turkish version of the Wijma Delivery Expectancy/Experience Questionnaire (W-DEQ). ${ }^{13}$ The W-DEQ is a selfreport instrument designed to measure the fear of childbirth in terms of the woman's cognitive appraisal of childbirth. ${ }^{14}$ The W-DEQ was developed to measure the construct of fear of childbirth both during pregnancy (version A) and after delivery (version B). Each scale of the W-DEQ is a 33-item self-assessment rating scale, and each item ranges from "not at all" (score 0) to "extremely" (score 5). The sum score may thus range from 0 to 165 , and fear of childbirth was defined as W-DEQ sum score of $\geq 85$.

Participants in both groups also completed the Beck Depression Inventory $(\mathrm{BDI})^{15}$ which is a 21-item questionnaire frequently used to measure depression in patients with chronic pain. For the general population, a score of 21 or over represents depression. For people who have been clinically diagnosed, scores from 0 to 9 represent minimal depressive symptoms, scores of 10 to 16 indicate mild depression, scores of 17 to 29 indicate moderate depression, and scores of 30 to 63 indicate severe depression.

One blinded examiner included the patients in the study and collected the completed inquiry forms. Diagnosis of FS and analysis of WPI and SS scores was made by one examiner who was not blinded. All other analyses, based on VAS, FIQ, STAI, W-DEQ, and BDI, were evaluated by another examiner who was blinded to the patients and controls.

\section{Statistical analysis}

Power analyses were performed by G*Power version 3.1.8. The power of this study was calculated for one of the primer outcomes of the study which named the VAS score. Ninety-seven patients for each group [FS(+) and FS(-)] achieve $80 \%$ power to detect a difference of " 1.0 " unit between two group means with estimated group standard deviations of "2.0" with a significance level (alpha) of 0.05 using two sided two sample t-test.

All other statistical computations, comparisons, and analyses were performed using SPSS version 15.0 for Windows, (SPSS Inc., Chicago, IL, USA). Descriptive statistics were presented as percentage or median (minimum-maximum). Differences between groups for categorical variables were analyzed by Chi-square test or Fisher's exact test, when appropriate. Degree of association between continuous variables was calculated by Spearman correlation. Stepwise linear regression

\begin{tabular}{|c|c|c|c|c|c|c|c|c|c|}
\hline & \multicolumn{4}{|c|}{ Fibromyalgia syndrome $(-)(\mathrm{n}=224)$} & \multicolumn{4}{|c|}{ Fibromyalgia syndrome $(+)(\mathrm{n}=136)$} & \multirow[b]{2}{*}{$p$} \\
\hline & $\mathrm{n}$ & $\%$ & Median & Min-Max & $\mathrm{n}$ & $\%$ & Median & Min-Max & \\
\hline Age (year) & & & 27 & $19-41$ & & & 26.5 & $20-42$ & 0.364 \\
\hline Body mass index & & & 27.05 & $19.02-45.33$ & & & 26.79 & 20.01-41.64 & 0.835 \\
\hline Gestational age (week) & & & 32 & $24-40$ & & & 32 & $21-39$ & 0.870 \\
\hline Satisfaction with marriage & & & 6 & $0-10$ & & & 7 & $3-10$ & 0.665 \\
\hline Number of healthy birth & & & 2 & $0-5$ & & & 2 & $0-5$ & 0.276 \\
\hline Number of abortus & & & 0 & $0-2$ & & & 0 & $0-2$ & 0.158 \\
\hline Smoking habit & 55 & 28.7 & & & 40 & 24.2 & & & 0.399 \\
\hline Alcohol consumption & 24 & 12.4 & & & 22 & 13.3 & & & 0.965 \\
\hline
\end{tabular}


analyses were conducted to identify what factors contributed to SS and physical impairment scores. $P$ values less than 0.05 were considered statistically significant.

\section{RESULTS}

Univariate analysis of the differences between the FS and control groups showed no statistically significant differences between the groups in terms of mean age, body mass index, gestational age, satisfaction with marriage, smoking or alcohol abuse, and number of healthy births or abortus ( $p>0.05)$ (Table 1).

The most painful sites in both groups according to WPI and WPI scores of the groups are shown in Table 2. A statistically significant difference was found between the groups with respect to WPI score $(p<0.001)$, the mean WPI score being higher in FS group. Lower back, upper leg, lower leg, and abdomen were the most affected sites in FS group while abdomen, lower back, and hips were the most affected sites in control group. Compared to the FS group; jaw, shoulder

Table 2. Widespread pain index and symptom severity scores, and rates of painful area in groups

\begin{tabular}{|c|c|c|c|c|c|c|c|c|}
\hline & \multicolumn{4}{|c|}{ Fibromyalgia syndrome (-) (n=224) } & \multicolumn{4}{|c|}{ Fibromyalgia syndrome $(+)(n=136)$} \\
\hline & $\mathrm{n}$ & $\%$ & Median & Min-Max & $\mathrm{n}$ & $\%$ & Median & Min-Max \\
\hline Widespread Pain Index* & & & 1 & $0-6$ & & & 8 & $3-19$ \\
\hline Symptom Severity score* & & & 0 & $0-8$ & & & 6 & $5-10$ \\
\hline Fatigue* & & & 0 & $0-3$ & & & 3 & $1-3$ \\
\hline Waking unrefreshed* & & & 0 & $0-2$ & & & 2 & $0-3$ \\
\hline Cognitive symptoms* & & & 0 & $0-3$ & & & 2 & $0-3$ \\
\hline \multicolumn{9}{|l|}{ Affected site ${ }^{*}$} \\
\hline Shoulder girdle (left)* & 17 & 7.5 & & & 43 & 31.6 & & \\
\hline Shoulder girdle (right)* & 7 & 3.1 & & & 35 & 25.7 & & \\
\hline Upper arm (left)* & 9 & 4.0 & & & 37 & 27.0 & & \\
\hline Upper arm (right)* & 6 & 2.6 & & & 45 & 33.0 & & \\
\hline Lower arm (left)* & 5 & 2.2 & & & 30 & 22.0 & & \\
\hline Lower arm (right)* & 4 & 1.7 & & & 22 & 16.1 & & \\
\hline Hip (buttock, trochanter) (left) $\dagger$ & 56 & 25.0 & & & 44 & 32.3 & & \\
\hline Hip (buttock, trochanter) (right) $\dagger$ & 46 & 20.5 & & & 34 & 25.0 & & \\
\hline Upper leg (left)* & 19 & 8.4 & & & 63 & 46.3 & & \\
\hline Upper leg (right)* & 25 & 11.1 & & & 72 & 52.9 & & \\
\hline Lower leg (left)* & 22 & 9.8 & & & 75 & 55.1 & & \\
\hline Lower leg (right)* & 23 & 10.2 & & & 76 & 55.8 & & \\
\hline Jaw (left)* & 2 & 0.9 & & & 27 & 17.6 & & \\
\hline Jaw (right)* & 3 & 1.3 & & & 21 & 15.4 & & \\
\hline Chest* $^{*}$ & 11 & 4.9 & & & 45 & 33.0 & & \\
\hline Abdoment & 88 & 41.1 & & & 72 & 52.9 & & \\
\hline Upper back* & 14 & 6.5 & & & 58 & 42.6 & & \\
\hline Lower back $\dagger$ & 91 & 39.2 & & & 78 & 57.3 & & \\
\hline Neck $^{*}$ & 14 & 6.2 & & & 62 & 45.5 & & \\
\hline
\end{tabular}




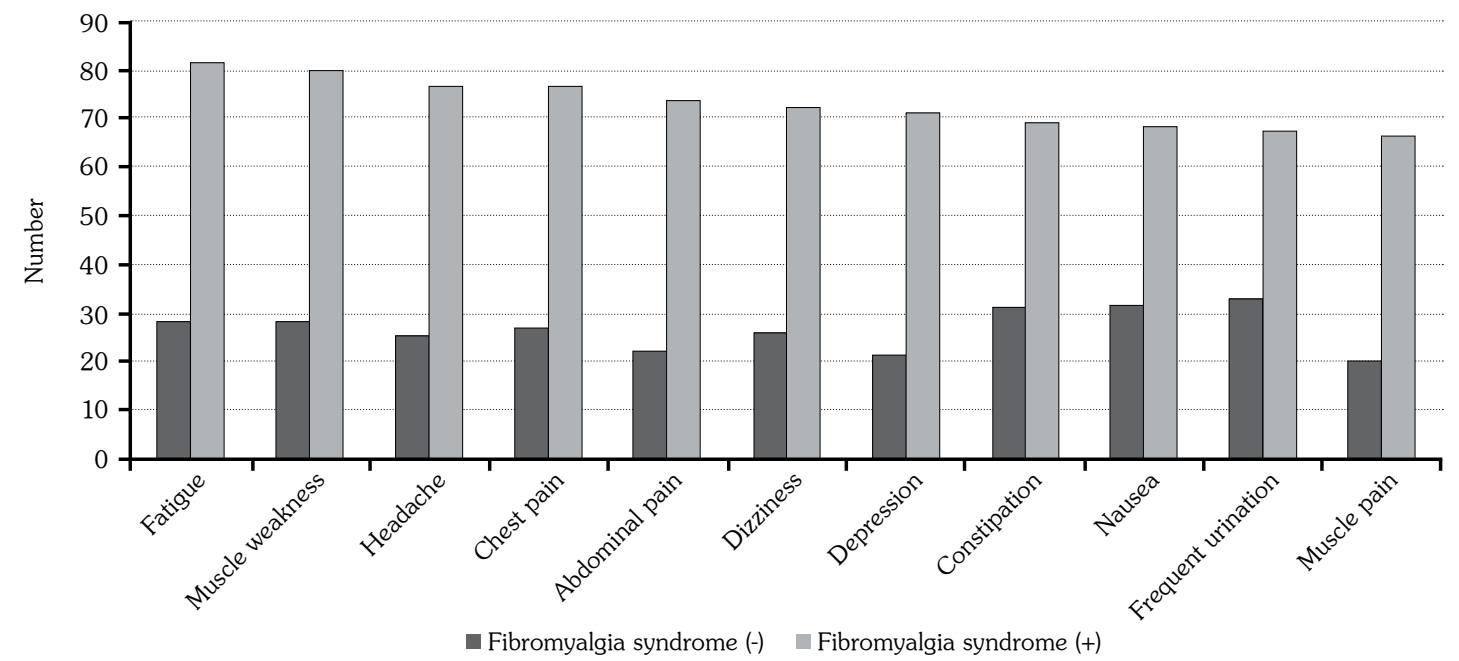

Figure 1. The most common symptoms of the Fibromyalgia syndrome in groups.

girdle, upper and lower arm, and neck were the less affected sites in control group. Compared to the control group, rates of painful areas were statistically significantly higher in the FS group $(p<0.001, p<0.01$, respectively).

Symptom severity scores of the groups are shown in Table 2. Compared to control group; mean SS, fatigue, waking unrefreshed, and cognitive symptoms values were statistically significantly higher in FS group $(p<0.001$ for all parameters).

Number of subjects with symptoms of FS in groups are shown in Figure 1. Fatigue/ tiredness, muscle weakness, headache, chest pain, pain/cramps in the abdomen, dizziness, depression, constipation, nausea, frequent urination, muscle pain were the most common somatic symptoms. Compared to control group, all of these symptoms were significantly higher in FS group ( $p<0.001$ for all parameters) (Figure 1).

Univariate analysis of the differences between the FS and control groups with respect to mean values of pain-VAS, physical affects (FIQ, PIS), and anxiety and depression levels are shown in Table 3. When the difference between FS and control groups in terms of these queries was studied as a single variable, it was shown statistically that pain scores increased and physical scores

Table 3. Univariate analysis of differences between fibromyalgia syndrome (+) and (-) groups with respect to pain intensity, physical affect, anxiety, fear of childbirth, and depression levels

\begin{tabular}{|c|c|c|c|c|c|}
\hline \multirow[b]{2}{*}{ Variables } & \multicolumn{2}{|c|}{ Fibromyalgia syndrome (-) $(n=224)$} & \multicolumn{2}{|c|}{ Fibromyalgia syndrome $(+)(n=136)$} & \multirow[b]{2}{*}{$p$} \\
\hline & Median & Min-Max & Median & Min-Max & \\
\hline Pain (Visual analog scale) & 1 & $0-10$ & 6 & $0-10$ & $<0.001$ \\
\hline Physical Impairment score & 1.18 & $0.00-2.30$ & 1.71 & $0.27-3.00$ & $<0.001$ \\
\hline Fibromyalgia Impact Questionnaire & 27.00 & $0.00-73.00$ & 72.00 & $13.00-99.00$ & $<0.001$ \\
\hline STAI state anxiety & 25 & $20-48$ & 52 & $25-76$ & $<0.001$ \\
\hline STAI trait anxiety & 22 & $20-46$ & 49 & $21-72$ & $<0.001$ \\
\hline W-DEQ vA & 32 & $0-64$ & 89 & $26-122$ & $<0.001$ \\
\hline W-DEQ vB & 30 & $0-62$ & 86 & $24-124$ & $<0.001$ \\
\hline Beck Depression Inventory & 6 & $0-27$ & 18 & $0-49$ & $<0.001$ \\
\hline
\end{tabular}




\begin{tabular}{|c|c|c|c|c|}
\hline \multirow[b]{2}{*}{ Characteristics } & \multicolumn{2}{|c|}{ Symptom Severity Score } & \multicolumn{2}{|c|}{ Physical Impairment Score } \\
\hline & $\mathrm{r}$ & $p$ & $\mathrm{r}$ & $p$ \\
\hline Age & 0.286 & 0.006 & 0.308 & $<0.01$ \\
\hline Body mass index & 0.013 & 0.773 & 0.026 & 0.642 \\
\hline Gestational age (week) & 0.230 & 0.087 & 0.298 & $<0.01$ \\
\hline Number of healthy birth & -0.017 & 0.813 & -0.041 & 0.561 \\
\hline Number of abortus & 0.014 & 0.818 & 0.049 & 0.611 \\
\hline Pain (Visual analog scale) & 0.617 & $<0.001$ & 0.667 & $<0.001$ \\
\hline STAI state anxiety & 0.577 & $<0.001$ & 0.521 & $<0.001$ \\
\hline STAI trait anxiety & 0.486 & $<0.001$ & 0.483 & $<0.001$ \\
\hline W-DEQ vA & 0.395 & $<0.001$ & 0.310 & $<0.001$ \\
\hline W-DEQ vB & 0.331 & $<0.001$ & 0.398 & $<0.001$ \\
\hline Beck Depression Inventory & 0.602 & $<0.001$ & 0.624 & $<0.001$ \\
\hline Fatigue & 0.402 & $<0.001$ & 0.414 & $<0.001$ \\
\hline Waking unrefreshed & 0.386 & $<0.01$ & 0.381 & $<0.01$ \\
\hline Cognitive symptoms & 0.381 & $<0.01$ & 0.377 & $<0.01$ \\
\hline Low back pain & 0.467 & $<0.001$ & 0.411 & $<0.001$ \\
\hline
\end{tabular}

were negatively affected in the presence of FS. FS group had higher values of pain $(p<0.001)$ and higher levels of physical impairment according to PIS and FIQ ( $<<0.001$ and $p<0.001$, respectively). Psychological scores were also negatively affected statistically in the presence of FS. FS group had higher values of state and trait anxiety evaluated with STAI $(p<0.001$ and $p<0.001$, respectively) and had higher degree of fear of childbirth both during pregnancy and after delivery evaluated with W-DEQ version $A$ and $B(p<0.001$ and $p<0.001$, respectively). FS group also had higher values of depression evaluated with BDI $(p<0.001)$.

The correlation analysis between SS and PIS with other demographic and clinical parameters are shown in Table 4 . Both the SS and physical impairment scores were significantly correlated with increased pain, depression, anxiety, fear of childbirth, fatigue, increased scores of waking unrefreshed, cognitive symptoms and increased rate of LBP $(p<0.001$ for all values). Physical impairment score was also significantly correlated with older age and advanced gestational age $(p<0.001)$.
Stepwise linear regression analysis was conducted to identify factors contributing to SS and physical impairment. Models from regression analysis for SS and physical impairment are shown in Table 5. Regression analysis showed that increased pain severity $\left(\mathrm{R}^{2}=0.31\right)$, increased STAI state anxiety scores $\left(\mathrm{R}^{2}=0.09\right)$, increased STAI trait anxiety scores $\left(\mathrm{R}^{2}=0.03\right)$, and increased level of depression level $\left(\mathrm{R}^{2}=0.02\right)$ were associated with higher scores of SS. A total of $45 \%$ variance was explained with these variables in SS (Table 6). Regression analysis showed that increased pain severity $\left(R^{2}=0.27\right)$, increased STAI trait anxiety scores $\left(\mathrm{R}^{2}=0.03\right)$, increased BDI $\left(\mathrm{R}^{2}=0.02\right)$ and increased level of depression level $\left(R^{2}=0.03\right)$ were associated with higher scores of physical impairment (Table 5). A total of 35\% variance was explained with these variables in physical impairment (Table 6).

\section{DISCUSSION}

In this study, we aimed to investigate the frequency and most common symptoms of fibromyalgia 


\begin{tabular}{|lccc|}
\hline \multicolumn{4}{|l}{ Table 5. Models from regression analysis for symptom severity and physical function } \\
\hline Model & $\mathrm{R}^{2}$ & Adjusted $\mathrm{R}^{2}$ & Factors added \\
\hline Symptom severity & & & \\
1 & 0.317 & 0.311 & Pain (Visual analog scale) \\
2 & 0.419 & 0.408 & STAI state anxiety \\
3 & 0.455 & 0.438 & STAI trait anxiety \\
4 & 0.473 & 0.452 & Beck Depression Inventory \\
Pysical Impairment Score & & & \\
1 & 0.295 & 0.275 & Pain (Visual analog scale) \\
2 & 0.315 & 0.308 & STAI trait anxiety \\
3 & 0.355 & 0.328 & Beck Depression Inventory \\
4 & 0.372 & 0.351 & Fatigue \\
\hline STAI: State-Trait Anxiety Inventory. & & & \\
\hline
\end{tabular}

among pregnant females and to determine the impacts of FS on physical functioning and psychological status. We found that, of a total of 360 pregnant subjects, $37.7 \%$ had FS. Furthermore, we observed that, in the presence of fibromyalgia, the physical and psychological parameters were negatively affected. Compared with pregnant females without FS, the number of painful areas was significantly higher in pregnant females with FS. Lower back, upper leg, lower leg, and abdomen were the most affected sites. Additionally, mean SS, fatigue, waking unrefreshed, and cognitive symptoms values were significantly higher in pregnant females with FS while fatigue/tiredness, muscle weakness, headache, chest pain, pain/cramps in the abdomen, dizziness, depression, constipation, nausea, frequent urination, and muscle pain were the most common somatic symptoms.

Fibromyalgia syndrome is a chronic pain disorder frequently affecting females of fertile age. ${ }^{6}$ The true incidence and prevalence of fibromyalgia are unknown. There is an overall 6 to $15 \%$ prevalence rate in the United States

\begin{tabular}{|lcccc|}
$\begin{array}{l}\text { Table 6. Results of stepwise multiple regression analysis to predict symptom severity and } \\
\text { physical impairment }\end{array}$ & Regression coefficient & $p$ & \multicolumn{2}{l}{$95 \%$ Confidence interval } \\
\cline { 3 - 5 } Characteristics & & & Lower limit & Upper limit \\
\hline Symptom severity score & 0.495 & $<0.001$ & 0.171 & 0.563 \\
Pain (Visual anolog scale) & 0.283 & 0.006 & 0.081 & 0.485 \\
STAI state anxiety & 0.385 & $<0.001$ & 0.167 & 0.510 \\
STAI trait anxiety & 0.684 & $<0.001$ & 0.205 & 0.716 \\
Beck Deppression Inventory & & & & 0.528 \\
Physical impairment & 0.410 & $<0.001$ & 0.143 & 0.333 \\
Pain (Visual anolog scale) & 0.224 & 0.005 & 0.197 & 0.695 \\
STAI trait anxiety & 0.617 & $<0.001$ & 0.210 & 0.702 \\
Beck Deppression Inventory & 0542 & $<0.001$ & 0.202 & \\
Fatigue & & & & \\
\hline STAI: State-Trait Anxiety Inventory. & & & & \\
\hline
\end{tabular}


with a five times greater incidence among females than males. In rheumatology clinics, the rate of new diagnosis is approximately 10 to $20 \%$, whereas in non-specialized settings, the rate is 2.1 to $5.7 \%{ }^{16,17}$ Prevalence of FS is reported as $3.6 \%$ in Turkish females aged between 20 and 64. ${ }^{18}$ Although FS frequently affects females of fertile age, the prevalence of fibromyalgia among pregnant females has not been clearly defined. In a study by Saa'd et al., ${ }^{5}$ out of 100 pregnant females recruited, 27 fulfilled FS criteria, compared to 136 subjects (37.7\%) out of 360 pregnant females in our study. The high rate of FS in our study may be due to the high number of participants. To our knowledge, no other prevalence studies have been reported in the literature. So, further studies are required to investigate the true prevalence of FS in pregnancy.

Similar to Saa'd et al.'s study results, ${ }^{5}$ in this study, the frequency of FS in pregnant females was higher. This result may be related to several mechanisms. Pregnancy related hormonal alterations might be one of these mechanisms. The fluctuations in ovarian hormones may be more related to changes in pain, painful symptoms, or pain sensitivity. Hormonal changes cause a laxity or loosening of ligaments and joints throughout the body. ${ }^{19}$ However, the association between the hormonal alterations in pregnancy and FS has not been investigated enough. In a study by Ostensen et al., ${ }^{6}$ it was reported that hormonal changes connected with abortion, use of hormonal contraceptives, and breast feeding did not modulate SS in the majority of patients with FS. According to Unruh, ${ }^{20}$ the interactions of female gonadal hormones with the many pathways involved in pain transmission are complex and occur at multiple levels, including primary afferents and neuromodulator systems. Because of the range and multiplicity of these effects, potential mechanisms underlying ovarian hormonal influence on pain perception or neural activity have been difficult to elucidate. Neuroendocrine changes may be another mechanism. Although the etiology of FS remains unclear, characteristic alterations in the pattern of sleep and changes in neuroendocrine transmitters such as serotonin, substance $P$, growth hormone, and cortisol suggest that regulation of the autonomic and neuro-endocrine system appears to be the basis of the syndrome. ${ }^{17}$ Human placenta is an endocrine organ, since it produces and releases a number of signaling substances including cytokines, neuropeptides, neurosteroids, and amines. ${ }^{21}$ So, placental neurohormones may be responsible for the high rate of FS in pregnancy. Psychiatric aspects may be another mechanism. Numerous studies report on comorbidity of FS and psychiatric disorders. Hypotheses about the link between depression and chronic pain include the notion that one causes the other or that shared pathogenetic mechanisms cause susceptible individuals to develop both conditions. ${ }^{22}$ High rates of anxiety, depression, and fear of childbirth in our patients with FS supported this possibility. Another mechanism may be central sensitization. One of the properties of this mechanism is the changes in sensory stimulation, presenting with low threshold values for pain, pressure, and temperature. ${ }^{23}$ Pregnancy related alterations in sensory perception thresholds and pain thresholds were reported in several studies. A significant reduction was demonstrated in sensory perception threshold and pain threshold between the prepartum and postpartum states. $^{24,25}$ On the contrary, some other authors reported no modifications in pain threshold during delivery. ${ }^{26,27}$ In these studies, tests were performed before and immediately after delivery. So, their results reflect only the parturition period and not the entire pregnancy period. We thought that neuroendocrine factors could change the pain threshold of pregnant females and make them prone to central sensitization. Therefore, females suffering from central sensitization may be more prone to developing FS. Reduced serotonin levels may be responsible for this situation. Further studies are needed investigating pain threshold during entire pregnancy period and the effects of neurohormones such as serotonin. Moreover, other several mechanisms, such as dysfunction of the central and autonomic nervous systems, immune system, and external stressors may be responsible for the high rate of FS in pregnancy.

Compared with pregnant females without FS; lower back, upper leg, lower leg, and abdomen were the most affected sites in our pregnant subjects with FS. These complaints are also complaints that may occur during pregnancy. LBP is a common complaint amongst females during pregnancy, having a great impact on their quality of life. Pregnancy-related pelvic girdle pain and pregnancy-related low back pain are 
two different patterns of LBP during pregnancy, although, a small group of females suffer from combined pain. Pelvic girdle pain and pregnancyrelated LBP are reported as common complaints among pregnant females. Both conditions are very common, with around $45 \%$ of all pregnant females suffering from pelvic girdle pain and/or pregnancy-related LBP. This pain can have an adverse impact on the quality of life for females who are affected..$^{28}$ LBP during pregnancy may be the result of mechanical, hormonal, and other factors, associated with the changes of the body. The literature clearly indicates that LBP may be disabling, limiting daily activities and impacting productivity and thus should not be ignored or left untreated. ${ }^{29}$ Consistent with the literature, lower back was the most affected region in both groups of our study population. However, compared to pregnant females without FS, the rate of LBP was significantly higher in pregnant females with FS (39.2\% and 57.3\%, respectively) as well as other painful areas. We also found a significant correlation between PIS and increased rate of low back pain. We thought that the existence of LBP might have serious negative effects on physical functioning in pregnant females with FS. Additionally, we observed that abdomen was one of the most affected part of the body in both groups. Numerous medical, surgical, psychiatric, gynecologic, and obstetric disorders can cause abdominal pain during pregnancy, some of which may be serious. ${ }^{30}$ Compared to pregnant females without FS, the rate of abdominal pain was significantly higher in pregnant females with FS. Pain/cramps in the abdomen was one of the most common somatic symptoms in this group as well. We suggested that abdominal pain could be a part of the symptoms group of FS in pregnant females if other causes could be ruled out. We also observed that, compared with the pregnant females with FS; jaw, shoulder girdle, upper and lower arm, and neck were the less affected sites in pregnant females without FS. These results suggested to us that pain in these regions most likely occurred due to fibromyalgia.

Being pregnant is accompanied by several physiological and biomechanical changes and with possible subsequent pregnancy complaints. The changes may also affect psychological health, including quality of life and well-being. ${ }^{31}$ Dørheim et al. $^{32}$ reported that pelvic girdle pain and fatigue/sleep problems were the main reasons given for sick leave. In our study, fatigue was the most prominent symptom in SS score of pregnant females with FS. It was also the most common somatic symptom. We found that fatigue is correlated with higher levels of SS and physical disability. Regression analysis showed that fatigue is one of the most common symptoms affecting physical functions in pregnant females with FS. So, we concluded that treating fatigue is essential in pregnant females for improving their quality of life.

Somatic symptoms are common in fibromyalgia. The most common somatic symptoms of FS in our FS group were fatigue/tiredness, muscle weakness, headache, chest pain, pain/cramps in the abdomen, dizziness, depression, constipation, nausea, frequent urination, and muscle pain. When we searched the literature on somatic symptoms of fibromyalgia in pregnancy, we could not find any other study on this subject. But, interestingly, we observed that majority of these symptoms were also among the most common complaints of pregnancy. Fatigue, problems with urination, constipation, heartburn, nosebleeds, swelling in legs, varicose veins, hemorrhoids, and breathing problems are reported as the most frequent symptoms of pregnancy. ${ }^{33} \mathrm{Zib}$ et al. ${ }^{34}$ reported that the third trimester is associated with the greatest number of symptoms. They reported a total of 38 symptoms occurring with a significantly different frequency in the third trimester. Most frequently reported symptoms were fatigue, pelvic pressure, insomnia, and lower backache in their pregnant subjects group. Furthermore, Geisser et al. ${ }^{35}$ investigated a potential pathologic mechanism of high frequency of somatic symptoms in their study. They created a composite measure of sensory sensitivity and compared this with measures of somatic symptoms, comorbid psychological disturbances, and selfreported physical functioning in 38 patients with FS and/or chronic fatigue syndrome. They concluded that sensory amplification may be an underlying pathophysiologic mechanism that is relatively independent of depression and depressive symptoms. As we stated before, a significant reduction was demonstrated in sensory perception threshold and pain threshold between the prepartum and postpartum states. ${ }^{24,25}$ So, altered sensory perception thresholds may be the 
reason for high prevalence of somatic symptoms. Moreover, considering the high rate of FS in pregnant subjects, it may be argued that the majority of the complaints of pregnancy may be the somatic symptoms of coexisting FS in these patients.

Pregnancy may be a vulnerable time period for females' psychosocial condition, with negative mood symptoms/depression frequently reported. Dunkel Schetter and Tanner ${ }^{36}$ reported that anxiety, depression, and stress in pregnancy are risk factors for adverse outcomes for mothers and children. Anxiety in pregnancy is associated with shorter gestation and has adverse implications for fetal neurodevelopment and child outcomes. Depressive symptoms in mothers during pregnancy are associated with lower birth weight infants with consequences for infant development. It was also reported in several studies that FS was related to increased anxiety and depression levels. ${ }^{37,38}$ It can be expected that anxiety and depression will be more frequent in coexistence of FS and pregnancy. Supporting this idea, we found that depression and anxiety were more frequent in pregnant females with FS as compared to pregnant females without FS. SS and physical function scores were found to be significantly correlated with increased depression and anxiety levels. The presence of anxiety and depression increased the SS and physical impairment of the patients. Storksen et al. ${ }^{39}$ reported that the presence of anxiety and depression increased the prevalence of fear of childbirth. We thought that increased anxiety and depression could lead to increased fear of childbirth in pregnant females with FS in our study population. So, psychiatric support is essential for pregnant females with FS to spend a healthy pregnancy and achieve good child health outcome.

Fibromyalgia syndrome is a disabling condition that may be difficult to assess and diagnose owing to its wide range of symptoms and common comorbidities. There is a statistically significant correlation between self-reported and objective measurements of disability for fibromyalgia patients. ${ }^{40}$ To our knowledge, this is the first study evaluating the effects of coexisting fibromyalgia on physical functions of pregnant females. In our study, we found that pregnant females with FS had higher levels of physical impairment. These results led us to conclude that the existence of fibromyalgia in pregnant females resulted in poor physical condition. Poor functional outcome can be expected in these patients, but follow-up studies are needed to establish this decision.

According to several studies, during pregnancy, maternal mental and physical wellbeing are transferred to the fetus resulting in epigenetic changes implicating consequences for a lifetime. ${ }^{41-43}$ Monk et al. ${ }^{41}$ reported that prenatal exposure to maternal stress, anxiety, and depression can have lasting effects on infant development with risk of psychopathology. The in utero environment is regulated by placental function and there is emerging evidence that the placenta is highly susceptible to maternal distress and a target of epigenetic dysregulation. So, with respect to the child's future development, factors leading to severe maternal stress and distress should be handled carefully. We observed that FS is common among pregnant subjects and the existence of FS in pregnancy is a severe factor for maternal stress, anxiety, and depression. We think that investigating the presence of fibromyalgia is essential for healthy pregnancy and good child health outcome.

The only limitation of this study is the lack of a follow-up period. The questions "what is the functional outcome of the FS patients who are pregnant" and "what is the child health outcome in pregnant subjects with fibromyalgia" may be better answered by follow-up studies including the delivery and post-partum period.

In conclusion, we demonstrated that fibromyalgia is quite common among pregnant females. Furthermore, we observed that, in the presence of fibromyalgia, physical and psychological parameters were negatively affected. The existence of widespread pain, severe complaints of pregnancy, increased levels of physical dysfunction, and poor psychological condition may be possible signs of FS in pregnancy. Therefore, therapeutic measures for FS should be well-established to support healthy pregnancy and good child health outcome.

\section{Declaration of conflicting interests}

The authors declared no conflicts of interest with respect to the authorship and/or publication of this article. 


\section{Funding}

The authors received no financial support for the research and/or authorship of this article.

\section{REFERENCES}

1. Adams K, Bombardier C, van der Heijde DM. Safety of pain therapy during pregnancy and lactation in patients with inflammatory arthritis: a systematic literature review. J Rheumatol Suppl 2012;90:59-61.

2. Mogren IM, Pohjanen AI. Low back pain and pelvic pain during pregnancy: prevalence and risk factors. Spine (Phila Pa 1976) 2005;30:983-91.

3. Clauw DJ. Musculoskeletal pain and dysfunction. fibromyalgia. In: Ruddy S, Haris ED, Sledge CB, editors. Keley's Textbook of Rheumatology. 6th ed. Philadelphia: WB Saunders Company; 2001. p. $417-28$.

4. Yunus MB. The role of gender in fibromyalgia syndrome. Curr Rheumatol Rep 2001;3:128-34.

5. Saa'd S, Many A, Jacob G, Ablin JN. High prevalence of fibromyalgia symptoms among healthy full-term pregnant women. Rheumatol Int 2013;33:1555-60.

6. Ostensen M, Rugelsjøen A, Wigers SH. The effect of reproductive events and alterations of sex hormone levels on the symptoms of fibromyalgia. Scand $\mathrm{J}$ Rheumatol 1997;26:355-60.

7. Wolfe F, Clauw DJ, Fitzcharles MA, Goldenberg DL, Katz RS, Mease P, et al. The American College of Rheumatology preliminary diagnostic criteria for fibromyalgia and measurement of symptom severity. Arthritis Care Res (Hoboken) 2010;62:600-10.

8. Sarmer S, Ergin S, Yavuzer G. The validity and reliability of the Turkish version of the Fibromyalgia Impact Questionnaire. Rheumatol Int 2000;20:9-12.

9. Burckhardt CS, Clark SR, Bennett RM. The fibromyalgia impact questionnaire: development and validation. J Rheumatol 1991;18:728-33.

10. Bennett R. The Fibromyalgia Impact Questionnaire (FIQ): a review of its development, current version, operating characteristics and uses. Clin Exp Rheumatol 2005;23:154-62.

11. Le Compte WA, Ömer N. Development of the Turkish edition of state-trait anxiety inventory. In: Spielberg CD, Diaz Guerro R, editors. Crosscultural anxiety. Washington DC: Hemisphere Publishing Co.; 1976. p. 51-68.

12. Spielberger CD. Theory and research on anxiety. New York: Academic Press; 1966. p. 17-86.

13. Korukcu O, Kukulu K, Firat MZ. The reliability and validity of the Turkish version of the Wijma Delivery Expectancy/Experience Questionnaire (W-DEQ) with pregnant women. J Psychiatr Ment Health Nurs 2012;19:193-202.

14. Wijma K, Wijma B, Zar M. Psychometric aspects of the W-DEQ; a new questionnaire for the measurement of fear of childbirth. J Psychosom Obstet Gynaecol 1998;19:84-97.

15. Beck AT, Steer RA, Garbin MG. Psychometric properties of the Beck Depression Inventory: twenty-five years of evaluation. Clin Psychol Rev 1988;8:77-100.

16. Alarcon GS. Fibromyalgia. In: Kopman WJ, Boulware DW, Heudebert GR, editors. Clinical primer of rheumatology. Philadelphia: Lipincot Williams and Wilkins; 2003 p. 226-35.

17. Jahan F, Nanji K, Qidwai W, Qasim R. Fibromyalgia syndrome: an overview of pathophysiology, diagnosis and management. Oman Med J 2012;27:192-5.

18. Topbas M, Cakirbay H, Gulec H, Akgol E, Ak I, Can G. The prevalence of fibromyalgia in women aged 20-64 in Turkey. Scand J Rheumatol 2005;34:140-4.

19. Casagrande D, Gugala Z, Clark SM, Lindsey RW. Low Back Pain and Pelvic Girdle Pain in Pregnancy. J Am Acad Orthop Surg 2015;23:539-49.

20. Unruh AM. Gender variations in clinical pain experience. Pain 1996;65:123-67.

21. De Bonis M, Torricelli M, Severi FM, Luisi S, De Leo V, Petraglia F. Neuroendocrine aspects of placenta and pregnancy. Gynecol Endocrinol 2012;28:22-6.

22. Arnold LM, Hudson JI, Keck PE, Auchenbach MB, Javaras KN, Hess EV. Comorbidity of fibromyalgia and psychiatric disorders. J Clin Psychiatry 2006;67:1219-25.

23. Marinus J, Van Hilten JJ. Clinical expression profiles of complex regional pain syndrome, fibromyalgia and a-specific repetitive strain injury: more common denominators than pain? Disabil Rehabil 2006 Mar;28:351-62.

24. Oshima M, Inagi T, Yokoyama K, Kikutani T, Sugimoto K, Shimada Y, et al. Pregnancy-related increases in sensory perception thresholds are not correlated with serum progesterone levels. Pain Pract 2003;3:120-4.

25. Ohel I, Walfisch A, Shitenberg D, Sheiner E, Hallak M. A rise in pain threshold during labor: a prospective clinical trial. Pain 2007;132:104-8.

26. Staikou C, Siafaka I, Petropoulos G, Katafigioti A, Fassoulaki A. Responses to mechanical and electrical stimuli are not attenuated by late pregnancy. Acta Anaesthesiol Belg 2006;57:277-81.

27. Whipple B, Josimovich JB, Komisaruk BR. Sensory thresholds during the antepartum, intrapartum and postpartum periods. Int J Nurs Stud 1990;27:213-21.

28. Vermani E, Mittal R, Weeks A. Pelvic girdle pain and low back pain in pregnancy: a review. Pain Pract 2010;10:60-71.

29. Katonis P, Kampouroglou A, Aggelopoulos A, Kakavelakis K, Lykoudis S, Makrigiannakis A, et al. Pregnancy-related low back pain. Hippokratia 2011;15:205-10.

30. Cappell MS, Friedel D. Abdominal pain during pregnancy. Gastroenterol Clin North Am 2003;32:1-58. 
31. Haakstad LA, Torset B, Bø K. What is the effect of regular group exercise on maternal psychological outcomes and common pregnancy complaints? An assessor blinded RCT. Midwifery 2016;32:81-6.

32. Dørheim SK, Bjorvatn B, Eberhard-Gran M. Sick leave during pregnancy: a longitudinal study of rates and risk factors in a Norwegian population. BJOG 2013;120:521-30.

33. Hark L, Catalano PM. Nutritional management during pregnancy. In: Gabbe SG, Niebyl JR, Simpson $\mathrm{JL}$, et al, editors. Obstetrics: Normal and Problem Pregnancies. 6th ed. Philadelphia: Elsevier Saunders; 2012. p. 125-139.

34. Zib M, Lim L, Walters WA. Symptoms during normal pregnancy: a prospective controlled study. Aust N Z J Obstet Gynaecol 1999;39:401-10.

35. Geisser ME, Strader Donnell C, Petzke F, Gracely RH, Clauw DJ, Williams DA. Comorbid somatic symptoms and functional status in patients with fibromyalgia and chronic fatigue syndrome: sensory amplification as a common mechanism. Psychosomatics 2008;49:235-42.

36. Dunkel Schetter C, Tanner L. Anxiety, depression and stress in pregnancy: implications for mothers, children, research, and practice. Curr Opin Psychiatry 2012;25:141-8.

37. Uçar M, Sarp Ü, Karaaslan Ö, Gül AI, Tanik N, Arik HO. Health anxiety and depression in patients with fibromyalgia syndrome. J Int Med Res
2015;43:679-85

38. Sener U, Ucok K, Ulasli AM, Genc A, Karabacak H, Coban NF, et al. Evaluation of health-related physical fitness parameters and association analysis with depression, anxiety, and quality of life in patients with fibromyalgia. Int J Rheum Dis 2016;19:763-72.

39. Storksen HT, Eberhard-Gran M, Garthus-Niegel S, Eskild A. Fear of childbirth; the relation to anxiety and depression. Acta Obstet Gynecol Scand 2012;91:237-42.

40. Segura-Jiménez V, Álvarez-Gallardo IC, Carbonell-Baeza A, Aparicio VA, Ortega FB, Casimiro AJ, et al. Fibromyalgia has a larger impact on physical health than on psychological health, yet both are markedly affected: the al-Ándalus project. Semin Arthritis Rheum 2015;44:563-70.

41. Monk C, Spicer J, Champagne FA. Linking prenatal maternal adversity to developmental outcomes in infants: the role of epigenetic pathways. Dev Psychopathol 2012;24:1361-76.

42. Urech C, Fink NS, Hoesli I, Wilhelm FH, Bitzer J, Alder J. Effects of relaxation on psychobiological wellbeing during pregnancy: a randomized controlled trial. Psychoneuroendocrinology 2010;35:1348-55.

43. Schitter AM, Nedeljkovic M, Baur $H$, Fleckenstein J, Raio L. Effects of Passive Hydrotherapy WATSU (WaterShiatsu) in the Third Trimester of Pregnancy: Results of a Controlled Pilot Study. Evid Based Complement Alternat Med 2015;2015:437650. 